\title{
CHAPITRE 1
}

\section{Introduction sur les fluides}

caloporteurs pour les réacteurs à neutrons rapides, une vue générale des problèmes scientifiques et techniques

Yves Bréchet, Robert Dautray et Jacques Friedel Membres de l'Académie des sciences 



\section{|Remarques préliminaires}

On pourrait dire, en forçant un peu le trait, que les réacteurs nucléaires sont en fait de grosses machines thermiques ayant une source de chaleur un peu particulière. Comme dans toutes les machines thermiques, il faut extraire la chaleur et la conduire vers une source froide où elle peut être transformée en travail mécanique via une turbine (à vapeur ${ }^{1}$ ou gaz), puis en énergie électrique. Décrit ainsi, le fonctionnement d'un réacteur nucléaire dépend de façon centrale de son fluide caloporteur. C'est au sujet de ce fluide caloporteur que le présent colloque a été organisé.

Mais il ne faut pas pour autant oublier que le caractère "particulier » de la source de chaleur, à savoir la réaction en chaîne de fission, impose à l'ingénieur des exigences très spécifiques qui compliquent singulièrement sa tâche.

- Tout d'abord, le fluide caloporteur "baigne » le combustible confiné dans sa gaine. II modifie le spectre neutronique et, selon que l'on veut travailler en spectre de neutrons thermiques ou en spectre de neutrons rapides, les choix du fluide caloporteur seront différents.

- Les centrales nucléaires comportent classiquement des barrières qui doivent prévenir le contact entre le combustible nucléaire et la biosphère : la gaine au contact du combustible, la cuve contenant le combustible et sa gaine, et enfin l'enceinte totale du réacteur. Et ce confinement de la radioactivité, requis par des exigences de sûreté, doit être rendu compatible avec l'extraction de chaleur : le fluide caloporteur doit extraire et transporter la chaleur sans pour autant laisser de chemin à la radioactivité. Cela impose par exemple que les éventuels produits de corrosion du circuit du fluide caloporteur, créés loin ou proches du cœur, adhèrent aux conduites et ne soient pas entraînés par le fluide vers les zones internes du cœur du réacteur, où ils seraient irradiés par les neutrons et deviendraient radioactifs. C'est pour satisfaire à ces contraintes contradictoires que trois barrières indépendantes, redondantes et distribuées loin l'une de l'autre dans l'ensemble de l'installation nucléaire, séparent les matériaux liés à la réaction de fission en chaîne et le générateur de vapeur couplé à la turbine et au condenseur. Elles ont été conçues suivant un cahier des charges qui exige qu'elles soient efficaces, non seulement pendant le régime nominal, mais aussi durant les arrêts pour maintenance, pour déchargement et durant les incidents ou accidents.

\footnotetext{
1 Par exemple, la turbine à vapeur d'eau de Phénix tournait à 3000 tours/minute. Les turbines à gaz pour le caloporteur sodium sont étudiées dans la diapositive 15 du document 2.1 .
} 
En effet, durant toutes ces opérations, (i) de la puissance nucléaire est continuellement émise par les noyaux radioactifs et, de plus, (ii) ces noyaux instables ont des demi-vies s'étalant des secondes et jours (iode131, demi-vie de 8 jours) jusqu'à une trentaine d'années pour n'aller que jusqu'à l'isotope 137 du césium.

- Le fluide caloporteur doit extraire la chaleur efficacement, ce qui conduit à essayer d'augmenter les températures et les pressions. Il doit le faire sans agresser de façon excessive le matériau qui le contient, ni du point de vue chimique, ni du point de vue de la sollicitation mécanique à chaud. Et enfin, il doit le faire sans risquer une transition de phase ${ }^{2}$ (ébullition) qui modifierait drastiquement et de façon incontrôlée les conditions de l'échange thermique aux parois.

Il est important de garder à l'esprit que l'ensemble des procédés liés aux échanges thermiques ou aux réactions chimiques du fluide caloporteur avec les matériaux, ou encore aux transferts de fluide, sont intrinsèquement hors d'équilibre et relèvent de la thermodynamique des phénomènes irréversibles. Cette discipline est souvent phénoménologique mais peut parfois être basée sur des approches au niveau atomique, ce qui leur donne un contenu physique mieux assuré (par exemple pour les comportements de fluide au seuil d'ébullition à la paroi).

C'est l'ensemble de ces exigences contradictoires qui doivent être présentes à l'esprit des ingénieurs, des scientifiques et des techniciens qui conçoivent les réacteurs dits de la quatrième génération. Mais les critères de choix de telle ou telle option, qui imposent une prise en compte globale du cycle du combustible, des conditions extérieures à la science et aux techniques, des exigences de non-prolifération et des aspects économiques. Elles sont beaucoup plus vastes que la simple question du fluide caloporteur et dépassent l'objectif de ce séminaire; de même que la question globale de la sécurité et de la sûreté du réacteur dans son ensemble. Nous nous focalisons ici sur la question spécifique des fluides caloporteurs et des conséquences scientifiques et technologiques des options de caloporteurs étudiées.

\footnotetext{
${ }^{2}$ La physicochimie des transitions de phase est exposée dans Thermodynamic Aspects of Phase Transitions (pp. 136-155 de [4]).
} 


\section{Encadré 1 - La crise d'ébullition}

La séparation entre une paroi chauffante et le fluide caloporteur n'est jamais une surface lisse parfaite. Les écoulements de fluide se produisent dans le volume bien sûr, mais aussi dans une couche limite d'épaisseur variable. Des bulles peuvent se former. Cette « ébullition nucléée » (bulles) est un processus de transfert de chaleur substantiel. Pourtant, si ces bulles coalescent près de la séparation fluide/solide, il peut se former un film continu de vapeur du fluide caloporteur. Comme la conductivité de la vapeur, aux mêmes température et pression, est très inférieure à celle du fluide, la température de la matière de surface du solide monte localement. On dit alors qu'il y a crise d'ébullition (boiling crisis). Les phénomènes intervenant dans le flux critique de chaleur et donnant lieu à la crise d'ébullition sont : passage de la convection naturelle à la germination de bulles de vapeur (nucleate ebullition), dépassement du flux de chaleur dit critique, formation d'un film quasi continu d'ébullition, montée très rapide de la température de la surface de la paroi (burn out of the heater), en fonction de la température de la surface d'échange, etc. Ces phénomènes sont décrits dans les diapositives 43, 44, 45 du document 6.1 et dans la référence [12]. Parmi les méthodes d'étude de ces phénomènes très rapides, mentionnons la mesure au point triple (vapeur, liquide, paroi) de l'angle apparent de contact à ce point triple entre la direction de la paroi et la direction de la séparatrice entre la vapeur et le liquide. Ces phénomènes sont hors d'équilibre (voir référence [14]) et relèvent donc de la thermodynamique hors d'équilibre (voir références [4, 12, 45]).

L'évaporation du fluide caloporteur nécessite de l'énergie pour séparer les molécules. Elle est ici fournie sous forme de chaleur, appelée enthalpie d'évaporation. L'appellation « chaleur latente » concerne une transformation à volume constant. De même, pour la chaleur sensible.

Il faut être attentif à ce que le changement en énergie interne $U$ n'égale pas l'énergie transférée comme chaleur quand le système est libre de varier son volume comme dans la piscine d'un RNR au sodium. Une partie de l'énergie cédée au système comme chaleur retourne à l'environnement comme travail d'expansion. Dans ce cas, l'énergie cédée au système à pression constante comme chaleur est égale à sa variation d'enthalpie. Celle-ci est la somme de la variation d'énergie interne $\Delta U$ et du travail p. $\Delta V$ (voir références $[4,44])$. La grande surface d'échange de chaleur en explique la taille (voir référence [44] pour la prise en compte des phénomènes d'évaporation - chaleur latente - et de condensation et, pour la surface d'échange de chaleur, voir infra encadré du paragraphe 1.4.1). Les valeurs de quelques-unes de ces enthalpies des fluides caloporteurs étudiées dans ce séminaire sont indiquées dans les diapositives 19 et 23 du document 5.1 pour les gaz, avec la notation $C_{p}$ ( $p=$ à pression constante) en $\mathrm{kJ} / \mathrm{kg}$. K (J pour joule, K pour kelvin), etc. 


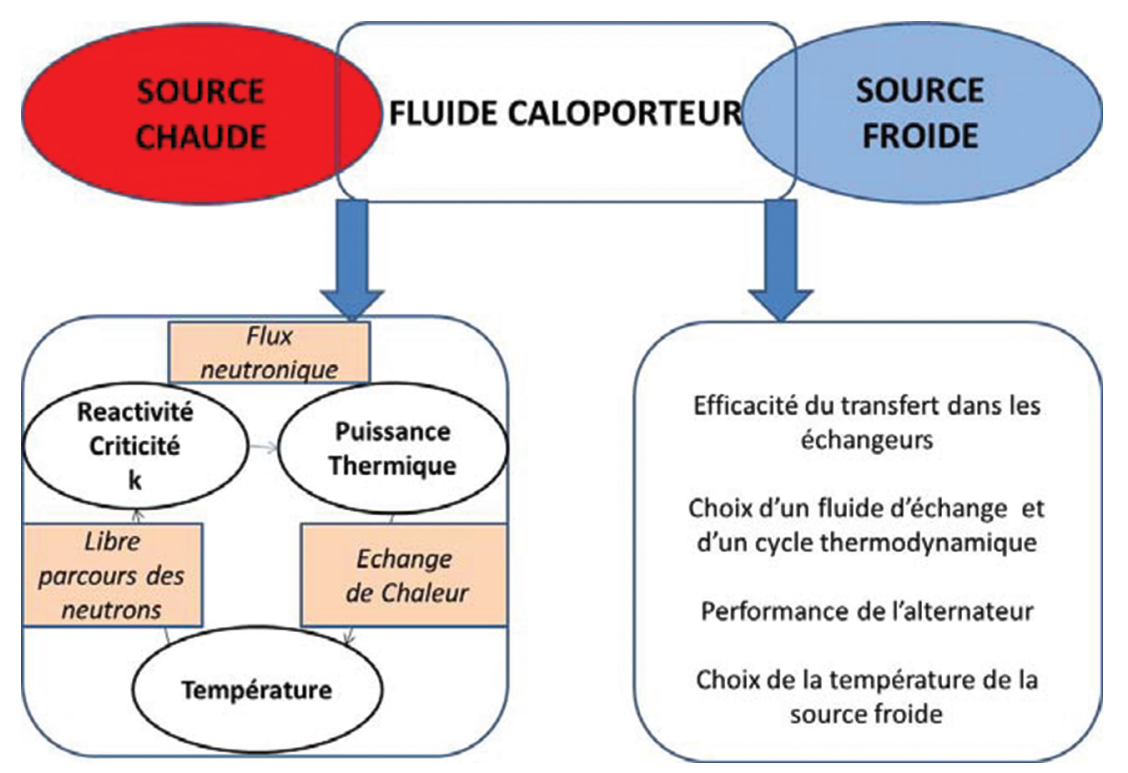

Figure 1.1

Positionnement logique du thème du séminaire dans une perspective thermodynamique des centrales.

Le positionnement logique du séminaire est schématisé sur la figure 1.1. La source chaude est le cœur du réacteur ; le fluide caloporteur a pour fonction d'extraire la chaleur et de la transporter jusqu'à la source froide où, via une machine thermique - que l'on peut caractériser comme étant la centrale entière, le générateur de vapeur, ou encore l'étage de conversion d'énergie thermique en travail thermodynamique -, elle sera transformée en travail puis, grâce à un alternateur, en électricité. Toutefois, nous y incluons toujours la source froide qu'est le condenseur et une source chaude qui peut être une condition aux limites. Le rôle du fluide caloporteur est essentiel pour deux raisons : (a) une raison directe qui est de nourrir le cycle thermodynamique de cette machine thermique aussi efficacement que possible; (b) une raison indirecte qui est son action sur la température du cœur. La réaction de fission en chaîne est la cause de la source de chaleur. Son efficacité dépend de l'économie de neutrons du cœur, laquelle est dépendante du libre parcours moyen des neutrons. La génération de chaleur résultant de ces réactions tend à réchauffer le cœur qui est refroidi par le fluide caloporteur. La température du cœur résulte de cet équilibre entre génération et extraction de chaleur. Cette température conduit à des modifications de la géométrie (dilatation, flambage) qui concernent le libre parcours moyen des neutrons, donc l'économie neutronique. En aval du cycle, le fluide caloporteur joue un rôle essentiel dans le fonctionnement de la machine thermique puisque c'est lui qui joue le rôle de source chaude. Plus la température chaude est élevée, plus 
le rendement thermodynamique sera élevé. Le choix des matériaux dans les échangeurs dépend fortement du fluide caloporteur et du fluide qui activera I'alternateur. Si l'on veut une machine thermique efficace, on doit choisir une source froide aussi froide que possible. En revanche, il peut être intéressant de générer en aval un fluide "chaud » (utilisable pour le chauffage) quitte à perdre en rendement électrique : on parle alors de " cogénération ».

Plus précisément, le cahier des charges proposé aux participants du séminaire était le suivant :

- faire le point des connaissances, des installations d'études, des logiciels, des incidents et accidents en ce qui concerne les caloporteurs et leurs circuits (surface intérieure, étanchéité, surfaces libres, corrosion, efforts mécaniques, physicochimie et chimie, etc.) dans les réacteurs à neutrons rapides, à partir de la paroi des sources chaudes, jusqu'au dispositif de conversion d'énergie calorifique en travail thermodynamique et de la source froide inclus ;

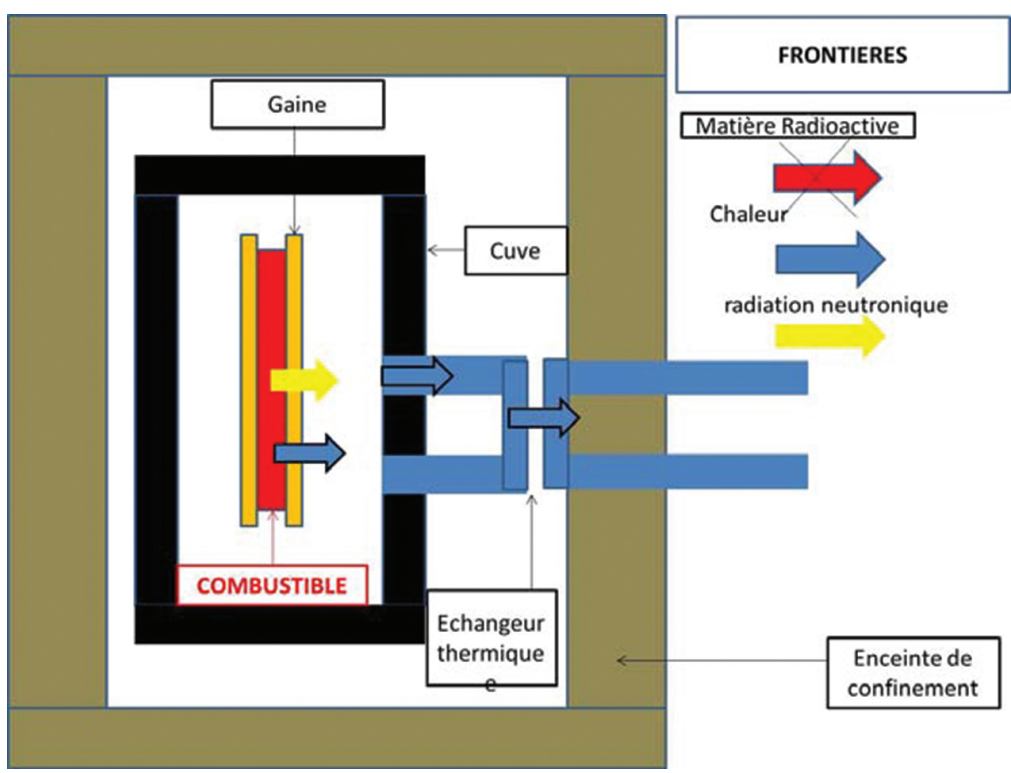

Figure 1.2

Les frontières dans un réacteur nucléaire et la « traversée » de ces frontières.

- étudier les propriétés de ces caloporteurs (physiques, chimiques, mécaniques, thermodynamiques, équilibres et physiques hors d'équilibre, changements de phase, etc.) tant dans les régimes nominaux de puissance que d'arrêt, de maintenance, de nettoyage, d'incident, d'accident, de sécurité, d'alerte, etc.) ; 
- sont concernées les installations passives (circuits, échangeurs de chaleur, filtres, etc.), actives (pompes ${ }^{3}$, vannes ${ }^{4}$, purgeurs, nettoyage, débouchage, etc.), I'instrumentation, la commande, le contrôle, les processus (maintenance, nettoyage, exploitation, manutention, transports...) ;

- préciser les verrous scientifiques, techniques, industriels et, si possible, les voies envisageables pour les traiter. Les calendriers associés doivent être explicités, ainsi que les ordonnancements des opérations ;

- les incertitudes doivent être, autant que faire se peut, intégrées dans un cadre exhaustif, cohérent, et replacées d'une manière relative et en arborescence des causes et des conséquences, avec les tentatives d'attribution à ces causes.

Le but du séminaire était de dresser à la fin une liste comparative des acquis et des verrous scientifiques et techniques, avec l'ordonnancement des travaux à exécuter pour en avancer la résolution, le tout étant comparé du point de vue des attraits pour la France et de ses atouts. Les différents exposés, tels qu'ils apparaissent dans le CD-Rom associé à ce livre et tels qu'ils sont référencés dans cette introduction, sont résumés dans le tableau 1.1.

\section{Tableau 1.1}

Les thèmes exposés au cours du séminaire.

\begin{tabular}{|c|c|}
\hline $\begin{array}{l}\text { 1. 1. Les différents fluides caloporteurs envisagés pour } \\
\text { les réacteurs rapides de la IV }{ }^{\ominus} \text { génération. }\end{array}$ & F. Carré (CEA) \\
\hline $\begin{array}{l}\text { 1.2. Les questions scientifiques et techniques dans le choix des flu- } \\
\text { ides caloporteurs. }\end{array}$ & $\begin{array}{l}\text { R. Dautray, Y. Bréchet } \\
\text { (Académie des sciences) }\end{array}$ \\
\hline 1.3. Le cahier des charges des réacteurs à neutrons rapides. & F. Gauché (CEA) \\
\hline $\begin{array}{l}\text { 2.1. Incidence du choix du sodium comme fluide caloporteur sur } \\
\text { la conception et le fonctionnement du réacteur. Contrôle préventif } \\
\text { des fuites et options possibles pour les échangeurs de chaleur. }\end{array}$ & G. Rodriguez (CEA) \\
\hline 2.2. Les propriétés du sodium et son comportement en cuve. & Ch. Latgé (CEA) \\
\hline 2.3. Retour d'expérience des RNR sodium. & J.-F. Sauvage (EDF) \\
\hline $\begin{array}{l}\text { 3.1. Le plomb fondu, comme fluide caloporteur pour les réacteurs } \\
\text { de la génération IV: problèmes spécifiques et comparaison avec } \\
\text { les autres fluides. }\end{array}$ & P. Agostini (ENEA) \\
\hline
\end{tabular}

${ }^{3}$ Par exemple, les pompes primaires (donc de sodium) ont des vitesses de rotation de 150 à 830 tours/minute et débitent environ 1000 kilogrammes de sodium/seconde (voir référence [2], p. 11).

${ }^{4}$ La robinetterie « sodium » est exposée pp. 232-234 de la référence [2]. 


\begin{tabular}{|c|c|}
\hline $\begin{array}{l}\text { 3.2. Technologie de mise en œuvre des fluides caloporteurs } \\
\text { lourds: plomb fondu et eutectique plomb-bismuth. }\end{array}$ & $\begin{array}{l}\text { V. Ulyanov } \\
\text { (IPPE Obninsk) }\end{array}$ \\
\hline $\begin{array}{l}\text { 3.3. La chimie du plomb et son interaction physicochimique avec } \\
\text { les matériaux de gainage et de tuyauteries. }\end{array}$ & T. Auger (ECP) \\
\hline $\begin{array}{l}\text { 4.1. Influence du choix des sels fondus sur la conception et } \\
\text { le fonctionnement des réacteurs MSR (Molten Salt Reactor). }\end{array}$ & D. Heuer (CNRS) \\
\hline $\begin{array}{l}\text { 4.2. Les caloporteurs de sels fondus: mise en œuvre dans } \\
\text { les réacteurs. }\end{array}$ & V. Ghetta (CNRS) \\
\hline $\begin{array}{l}\text { 5.1. Experimental feedback concerning the use of gases. Les gaz } \\
\text { et en particulier l'hélium comme fluides caloporteurs. }\end{array}$ & E. Bubelis (Karlsruhe IT) \\
\hline $\begin{array}{l}\text { 5.2. Interaction du fluide caloporteur hélium avec les matériaux, } \\
\text { spécificités des hautes températures. }\end{array}$ & C. Cabet (CEA) \\
\hline $\begin{array}{l}\text { 5.3. Incidence du choix du gaz, et en particulier de l'hélium, } \\
\text { comme fluide caloporteur sur la conception, le fonctionnement } \\
\text { et la sûreté des réacteurs. }\end{array}$ & J.-C. Garnier (CEA) \\
\hline $\begin{array}{l}\text { 6.1. Interaction des fluides avec les interfaces inertes. Sauts de } \\
\text { températures et de vitesses à l'interface : une vue microscopique, } \\
\text { conséquences sur la microfluidique et les échanges thermiques. }\end{array}$ & $\begin{array}{l}\text { E. Charlaix, J.-L. Barrat } \\
\text { (UJF Grenoble), } \\
\text { L. Boquet (ENS Lyon) }\end{array}$ \\
\hline $\begin{array}{l}\text { 6.2. Interaction des fluides avec des interfaces non inertes: le cas } \\
\text { de la fragilisation par les métaux liquides. }\end{array}$ & E. Glickman (Israël) \\
\hline $\begin{array}{l}\text { 7.1. Superphénix : fabrication et mise en œuvre des matériaux } \\
\text { de la cuve. }\end{array}$ & B. Escaravage (AREVA) \\
\hline $\begin{array}{l}\text { 7.2. Superphénix : fabrication des générateurs de vapeur, choix } \\
\text { du matériau des tubes d'échange. Inspection en service des tubes } \\
\text { d'échange et de la cuve. }\end{array}$ & $\begin{array}{l}\text { D. Buisine, } \\
\text { F. Champigny (EDF) }\end{array}$ \\
\hline 8.1. Seminar on Coolants for Fast Neutron Reactors. & $\begin{array}{l}\text { F. Carré (CEA), } \\
\text { Y. Bamberger (EDF) }\end{array}$ \\
\hline $\begin{array}{l}\text { 8.2. Coolant fluids for fat neutron reactors, scientific et technical } \\
\text { issues: Elements for a discussion. }\end{array}$ & $\begin{array}{l}\text { R. Dautray, Y. Bréchet } \\
\text { (Académie des sciences) }\end{array}$ \\
\hline
\end{tabular}

Après une série d'exposés introductifs (1.1 à 1.3), on trouvera donc successivement présentés le cas du sodium (2.1 à 2.3), du plomb et de ses alliages (3.1 à 3.3). Le cas des réacteurs à sels fondus est un peu particulier car, de tous les concepts de réacteurs, il est le seul qui ne découple pas le transfert de chaleur et celui de la matière radioactive puisque le fluide caloporteur est le combustible lui-même. Il est présenté dans les exposés 4.1 et 4.2 . Le cas des gaz, et plus spécifiquement de I'hélium comme fluide caloporteur, est présenté dans les exposés 5.1 à 5.3. Pour compléter cette vision scientifique et technologique des différentes options, les exposés 6.1 et 6.2 abordent la question fondamentale de l'interface fluide/solide, vue au niveau microscopique, qu'il s'agisse de problèmes de physique dans les interfaces non réactives (6.1) ou de problème de physicochimie comme en particulier dans 
le cas de la fragilisation par les métaux liquides (6.2). Les connaissances portant sur les caloporteurs sodium sont vastes et documentées, compte tenu des études, exploitations et installations d'essai, tant en France (Rapsodie, Phénix, Superphénix) qu'aux États-Unis, en Grande-Bretagne, Russie, Japon, Inde et Chine. Les deux derniers exposés portent sur la réalisation de la cuve de Superphénix, sur les choix de matériaux pour les générateurs de vapeur et les tubes d'échange, et sur les contrôles non destructifs. Il est de la plus haute importance que ces connaissances sur la réalisation concrète des réacteurs soient conservées et valorisées.

\section{Transfert de chaleur : de la source chaude à la source froide}

Le système caloporteur permet de transférer et de transformer une partie de l'énergie qui lie les noyaux des atomes en (i) énergie cinétique des fragments de fission, (ii) énergie cinétique des neutrons issus des fissions, (iii) neutrons issus des fragments de fission et (iv) énergie déposée par les rayons gamma émis par les fragments de fission et leurs descendants, etc. Ces diverses énergies cinétiques sont déposées sur leurs parcours dans la matière, pour la plus grande partie dans le combustible, sous forme de chaleur du milieu. Pour ce qui est dégagé dans le combustible, cette chaleur traverse les gaines (libre parcours des neutrons dans les gaines : voir figure 1.2 en page 15 ; épaisseur des gaines: $0,57 \mathrm{~mm}$, voir tableau 1.4 de la référence [5], p. 29) et chauffe par conduction le fluide caloporteur. Celui-ci contribuera à transférer, directement ou indirectement, une partie de cette chaleur au système de conversion d'énergie calorifique en travail thermodynamique et à la source froide, appelée condenseur (voir les six figures de la diapositive 13 du document 1.1).

Toutes ces fonctions du transfert de la chaleur vers un système de conversion d'énergie dépendent tant des lois de la physique, de la chimie, des matériaux accessibles, des processus physicochimiques possibles que des composants techniques qui les mettent en œuvre...

\section{1. Écoulement des fluides}

Les lieux d'exploitation de ces productions d'énergie, leur transport vers les zones de consommation, leur distribution, leur sécurité pour les humains, etc., dépendent des normes de protection des personnels et des populations concernés, des impacts sur les environnements et de l'évolution des besoins dus au changement climatique. Ceci comporte des incertitudes et entraîne 
des risques comme pour beaucoup de techniques. Ceux-ci doivent être évalués et gérés. Des compromis conduisent à diverses options (voir les six figures de la diapositive 13 du document 1.1).

Les critères de choix entre celles-ci comportent des bases scientifiques, techniques et industrielles.

Tous ces critères de choix varient dès aujourd'hui suivant les acteurs et sont appelés à être modifiés par les acteurs du futur. Après cinq décennies d'expériences (voir diapositives 4 et 5 du document 1.1), ceux des caloporteurs qui ont été retenus comme les plus susceptibles d'être capables, à divers avenirs, d'aboutir à des solutions robustes pour les différents sites et époques sont les suivants :

- le métal liquide 5 et notamment le sodium, le plomb et d'éventuels alliages (voir diapositives 11, 12, 14, 15, 16 du document 1.1) ;

- le gaz et notamment I'hélium (diapositives 9 et 10 du document 1.1);

- les sels fondus (voir diapositive 22 du document 1.1).

Les mérites respectifs des différents fluides, du point de vue du transfert de chaleur par unité de puissance de pompage, sont donnés dans le tableau 1.2.

Il est important de remarquer que les arguments donnés ci-dessus relèvent d'une approche macroscopique du transfert de quantité de mouvement et de chaleur. Se pose la question de définir les conditions aux interfaces (voir référence [31]), conditions de température, conditions de vitesse. II se trouve que, empiriquement, on constate que ces conditions aux limites dépendent de l'état de surface, de la rugosité et des conditions de mouillage. L'exposé 6.1 décline les situations dans lesquelles une approche à l'échelle atomistique peut s'avérer nécessaire pour rationaliser ces approches empiriques. La diapositive 4 de 6.1 donne les bases d'une description continue des conditions de transfert, introduisant la notion de "longueur de glissement», et la diapositive 12 synthétise l'effet de l'angle de movillage sur cette longueur, expérimentalement mesurée, calculée de façon analytique, ou par simulation en dynamique moléculaire. Le concept équivalent pour le transfert thermique est la «longueur de Kapitza » présentée dans la diapositive 27. Les apports potentiels des nanofluides, notamment en ce qui concerne la crise d'ébullition, découlent naturellement de ces analyses.

5 Voir la référence [34]. 
Tableau 1.2

Données physiques sur les différents fluides caloporteurs (densité $\rho$, capacité calorifique $C_{p}$ et viscosité dynamique $\mu$ ) et caractéristiques des différents réacteurs les utilisant. L'efficacité d'extraction est estimée par le rapport de la chaleur extraite $Q$ à la puissance de pompage $P$ nécessaire ${ }^{6}$.

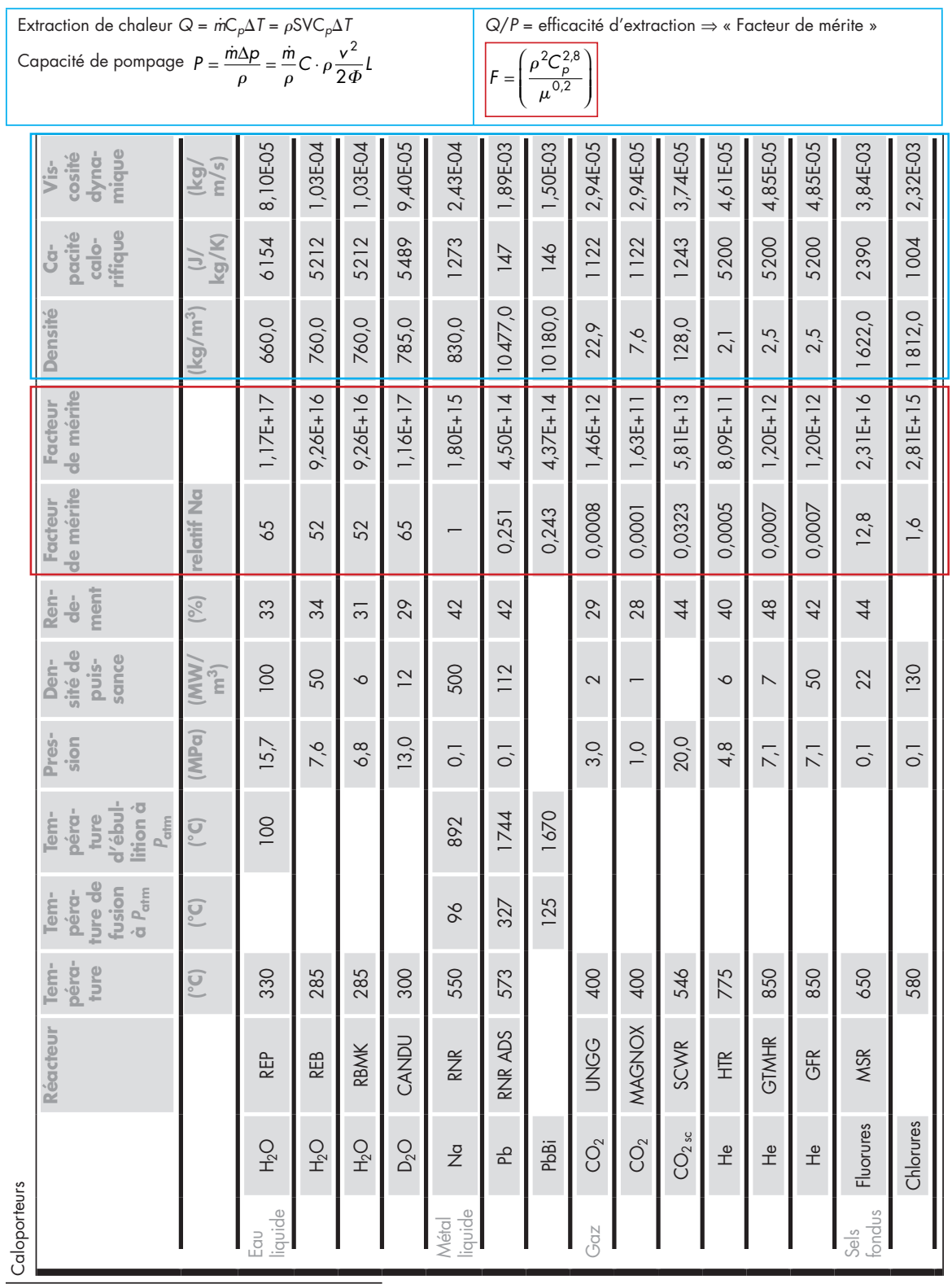

${ }^{6}$ La valeur absolue de ce "facteur de mérite » est donnée dans la colonne 11 et sa valeur rapportée à celle du sodium liquide dans la colonne 10 (H. Safa, communication privée, 2013). Cette puissance de pompage est calculée à partir de la chute de pression donnée par la théorie de Bernoulli, corrigée d'un coefficient $C$ traduisant la friction sur les parois, dépendant du nombre de Reynolds. $\Phi$ est le diamètre du tuyau et $L$ la hauteur. 


\subsection{Thermodynamique hors équilibre}

La fonction d'évacuation de la chaleur du cœur d'un réacteur nucléaire à neutrons rapides nécessite des spécificités additionnelles par rapport au cas des REP dit de III ${ }^{\text {e }}$ génération ${ }^{7}$ et des REB. Voir le chapitre 5 de [45].

En supprimant la fonction de modérateur, dans le cœur, il faut en particulier diminuer les taux de collisions élastiques et inélastiques des neutrons, et donc augmenter la proportion de matière fissile par unité de volume du cœur, donc le coût de l'investissement de ces matières fissiles par unité de volume. À flux de neutrons égal, cela augmenterait la puissance thermique par unité de volume du cœur. Pour compenser une partie des investissements supplémentaires par rapport aux REP, les concepteurs de RNR augmentent aussi le flux neutronique (passant de $4 \times 10^{14}$ neutrons $/ \mathrm{cm}^{2} \mathrm{~s}$ à $5 \times 10^{15} \mathrm{n} / \mathrm{cm}^{2} \mathrm{~s}$ ) donc, une seconde fois, la puissance thermique par unité de volume (la multipliant par environ 3, jusqu'à 300 à $400 \mathrm{~kW} / \mathrm{dm}^{3}$ ). Enfin, pour augmenter le rendement du cycle thermodynamique du système de conversion (voir diapositive 3 du document 2.1) de puissance thermique en travail thermodynamique $(0,34$ dans les REP), les concepteurs augmentent, autant que les matériaux (notamment ceux de la gaine du combustible) le permettent, la température de sortie du fluide caloporteur du cœur (passant de $350^{\circ} \mathrm{C}$ à $550^{\circ} \mathrm{C}$ quand on passe de l'eau au sodium) pour atteindre un rendement proche de 0,45-0,48.

Tous ces nombres sont portés dans les tableaux des communications 2.1, $3.1,4.1$ et 5.1 (voir diapositive 6 du document 2.1 pour une comparaison avec la thermodynamique des REP).

Ces augmentations de puissance locale dans le cœur du réacteur (par rapport aux REP) augmentent fortement la montée de température du fluide caloporteur lors de sa traversée du cœur (passant de une à deux dizaines de degrés Celsius à près de une à deux centaines de degrés Celsius - voir pour le sodium le tableau de la diapositive du document 2.1, pour la pression de vapeur de sodium la diapositive 27 du document 2.2 et pour la solubilité de diverses espèces physicochimiques dans le sodium les diapositives 8,9 et 10 du même document; pour le plomb la pression de vapeur saturée en fonction de la température est donnée par la diapositive 7 du document 3.1). $C^{\prime}$ est dire que les hétérogénéités de températures dans les réacteurs, donc les efforts mécaniques locaux subis, et ainsi les déformations et les propagations éventuelles de défauts, deviennent bien plus élevés que dans les REP. Et bien sûr, les flux neutroniques étant plus importants, les taux d'irradiations sont eux aussi plus élevés.

7 Un exemple en est l'EPR (European Power Reactor). 
Le chemin parcouru par un produit de fission est de l'ordre de $10^{-4} \mathrm{~cm}$, soit $1 \mu \mathrm{m}$ (en environ $10^{-12} /$ densité massique [secondes], voir référence [6], figure 5.3 , p. 115). L'énergie cinétique des fragments de fission est donc déposée dans la pastille de métal lourd.

\section{Encadré 2 - Libre parcours moyen et hétérogénéités des dépôts de chaleur}

Le libre parcours moyen de fission des neutrons naissant en moyenne avec une énergie cinétique de $2 \mathrm{MeV}$, dans l'oxyde de plutonium et d'uranium, est d'un ordre tel que :

$$
(1 / L) \mathrm{cm}=2 \text { barns }\left(10^{-24} \mathrm{~cm}^{2}\right) \times 4 \times 10^{22} \text { noyaux } / \mathrm{cm}^{3} \times 0,85=0,3 \mathrm{~mm} \text {, }
$$

où 2 barns est une section efficace moyenne (entre les différents noyaux, les différentes énergies des neutrons, les différentes réactions nucléaires - absorption, diffusion élastique, inélastique, fission, etc.) des réactions nucléaires neutrons-noyaux lourds et où 0,85 est le rapport des densités massiques de l'uranium métal et de l'oxyde d'uranium.

Les dimensions des pastilles étant d'environ $10 \mathrm{~mm}$, ces énergies cinétiques des neutrons issus d'une fission sont déposées, en majorité, dans le rayon de la pastille qui leur a donné naissance.

Au contraire, les rayons gamma de la fission et ceux des produits de fission ont des longueurs d'absorption bien supérieures aux dimensions des combustibles.

En résumé, le fluide caloporteur est chauffé essentiellement par :

$364(171+193=364)$ assemblages $\times 271$ aiguilles $^{8} \times 160 \mathrm{~cm} \times 0,85$ $\times 3,14=\mathbf{4 2 1} \mathbf{~ m}^{\mathbf{2}}$ de contact environ

(cas de Phénix : voir diapositives 3 à 11 du document 2.3, référence [1] J.F. Sauvage] et référence [2], pp. 83-90) avec les crayons qui sont, eux, à une température allant de $450{ }^{\circ} \mathrm{C}$ à $550{ }^{\circ} \mathrm{C}$, avec au centre des températures maximales de $1800{ }^{\circ} \mathrm{C}$ et des pressions venant des gaz de fission', des alpha, de l'oxygène des oxydes décomposés montant progressivement jusqu'à 90 bars, la pression du fluide caloporteur étant d'environ 1 bar = les pertes de charges, du même ordre de grandeur (voir diapositive 6 du document 1.2).

8 Plus précisément, le nombre d'aiguilles dans un réacteur comme Phénix est d'environ 180000 , celui de pastilles dans ces aiguilles est d'environ 12 millions (voir référence [2], p. 74). C'est dire l'importance des contrôles de qualité.

${ }^{9}$ La fraction des gaz de fission relâchés par les pastilles croît avec le taux de combustion (voir référence [2], figure p. 76). II atteint $70 \%$ (respectivement $80 \%$ ) pour un taux de combustion du métal lourd de $3 \%(6 \%)$. 


\section{Encadré 3 - Transmission de chaleur}

La surface d'échange de chaleur dans l'ensemble des six échangeurs de chaleur intermédiaires (voir référence [2], p. 84) et celle du générateur de vapeur et convertisseur d'énergie thermique en travail thermodynamique (voir chapitre 7 de la référence [2]) sont du même ordre de grandeur (457 $\mathrm{m}^{2}$ pour Phénix).

C'est parce que les surfaces d'échange de chaleur sont du même ordre de grandeur, tant pour gaine/fluide caloporteur, fluide caloporteur primaire ${ }^{10} /$ fluide caloporteur secondaire $^{11}$, fluide caloporteur secondaire/fluide d'alimentation de la turbine et des compresseurs, que les échangeurs de chaleur sont aussi volumineux que le réacteur nucléaire. Finalement, ce sont ces phénomènes de transfert de chaleur qui dimensionnent les composants les plus volumineux des centrales nucléaires RNR (voir les tours de refroidissement aéroréfrigérantes sur la figure de la diapositive 19 de 2.3). Un procédé permettant de les comparer est l'utilisation des nombres de Nusselt (convective heat transfer coefficient/conductive heat transfer coefficient) et du nombre de Sherwood, pour le transfert par matière. Ces nombres permettent aussi de caractériser les marges de convection libre (passive). Une indication de cette propriété est le " nombre de Grashof » défini et expliqué dans 3.1 (diapositive 7 ; et pour les propriétés physiques comparées entre le plomb et le sodium, les diapositives 5 et 7 de ce document 3.1). Le nombre de Grashof du plomb est environ 12 fois plus élevé que celui du sodium.

Figure 3 du document 1.2 :

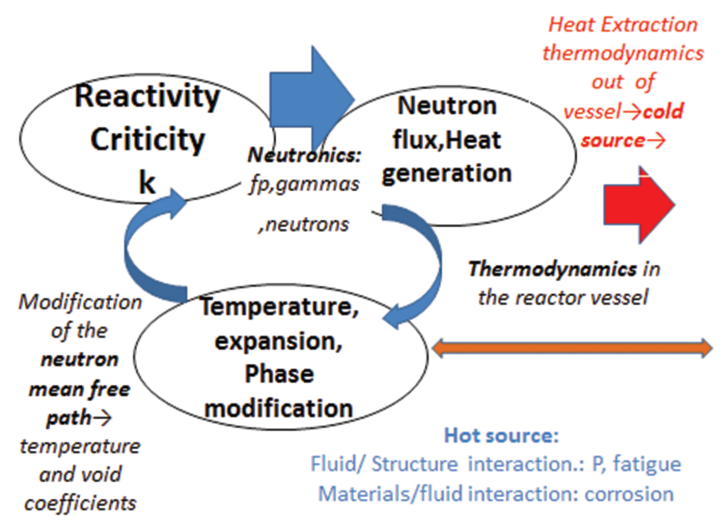

10 Un échangeur intermédiaire comporte environ 2300 tubes d'échange de chaleur.

11 Par exemple, dans Phénix, le débit de sodium dans le générateur de vapeur est de 700 kilogrammes/seconde alors que le débit d'eau à vaporiser est de 250 tonnes/heure. 


\section{3. Évacuation de la puissance, gradient de température et qualité de la puissance thermique}

\subsubsection{Surfaces méfalliques successives d'échange de chaleur}

Le taux de transfert de chaleur dimensionne tant les échangeurs de chaleur que la conversion d'énergie et aussi le cœur du réacteur.

Ces grandes surfaces d'échange, ces hautes températures, ces écoulements à grande vitesse (environ 10 mètres/seconde dans Phénix à comparer pour les autres fluides caloporteurs à 1-3 mètres/seconde dans les diapositives 5 à 11 du document 8.1) nécessitent de grandes quantités de tubes pour les échangeurs de chaleur et les générateurs de vapeur d'eau (357 tubes dans les échangeurs intermédiaires de Superphénix, voir les diapositives 2, 3, 4, 19 et 20 du document 7.2).

\subsubsection{Changement de phase}

Un autre trait distinctif des fluides caloporteurs des RNR concerne leurs éventuels changements de phase (voir, outre l'encadré "Crise d'ébullition », les diapositives 3 du document 1.1 et 5 du document 3.1).

Ceux-ci sont liés au triplet température locale-pression locale-densité massique locale et à la composition chimique ou physicochimique locale. En cas de décroissance de ces conditions, le fluide peut se solidifier localement. En cas d'augmentation « incidentelle » de la température locale dans le cœur du réacteur, les libres parcours des neutrons sont augmentés. Le spectre des énergies cinétiques des neutrons est donc déplacé vers les basses énergies. Les sections efficaces y sont plus fortes et la réactivité augmente. C'est l'effet positif de vide sur la réactivité des RNR.

Son articulation dans le triangle ${ }^{12}$ des contre-réactions participe à la stabilité des conditions thermodynamiques du cœur (voir diapositive 3 du document 1.2).

12 Le triangle $(a, b, c)$ des contre-réactions thermiques (dont l'agitation des noyaux) = effet Doppler (voir diapositive 3 du document 1.2 et référence [3], pp. 379-380 (The intrinsic safety)).

(a) Réactivité (neutronique) flux de neutrons. Flux de neutrons = puissance thermique locale.

(b) Puissance thermique dans le cœur (thermodynamique du cœur) température.

(c) Densité massique (dilatation, déformation, libres parcours moyens) réactivité. 
Parmi les conditions thermodynamiques ${ }^{13}$ nécessaires pour rester dans une gamme (température, densité de masse, pression) sans changement de phase, il nous faut insister sur le rôle privilégié de la pression locale dans tout le système des caloporteurs, depuis les gaines ${ }^{14}$ jusqu'au générateur de travail thermodynamique inclus (voir référence [2], tableau p. 93 et figure p. 97).

Ces pressions dans le système caloporteur sont liées aux températures de ces fluides (dans les circuits primaires, secondaires, les générateurs de vapeur $^{15}$, etc.). Voir ces températures locales dans les diapositives 13 et 15 du document 4.1 .

Ces températures des fluides résultent aussi de celles des gaines et de leur conductivité thermique, des températures d'entrée dans le cœur et des vitesses des fluides caloporteurs. Celles-ci et celles-là sont données dans les diapositives 5 et 8 du document 5.3 dans le cas d'un caloporteur gazeux. Les phénomènes de variations rapides des vitesses de fluides caloporteurs et de températures aux interfaces sont aussi à prendre en compte (voir la diapositive 37 du document 6.1).

Pour les fluides caloporteurs qui induisent des différences de pression, les conséquences sur les efforts mécaniques et les déformations locales sont considérables. Notons que les centrales de production d'énergie ont vécu avec cette contrainte pour la quasi-totalité des productions, tant en ce qui concerne les centrales nucléaires, depuis un demi-siècle, que pour toutes les machines à vapeur depuis trois siècles.

Un exemple de phénomène extrême peut se produire localement et même gagner des parties substantielles du circuit du fluide caloporteur. Un spécimen en est le refroidissement du plomb et sa solidification d'abord locale, mais bloquant ensuite la circulation d'un des circuits de caloporteurs. Ce cas a été étudié, par exemple pour le plomb bismuth dans la diapositive $35 \mathrm{du}$ document 3.1.

\footnotetext{
${ }^{13}$ Parmi les propriétés thermodynamiques, donnons quelques éléments pour le sodium : masse volumique à $20{ }^{\circ} \mathrm{C}=0,971 \mathrm{~g} / \mathrm{cm}^{3}$; état d'oxydation : +1 ; oxyde : base forte ; point de fusion $=97,80{ }^{\circ} \mathrm{C}$; point d'ébullition $=883{ }^{\circ} \mathrm{C}$; énergie de fusion $=2,598 \mathrm{~kJ} / \mathrm{mol}$; énergie de vaporisation $=96,96 \mathrm{~kJ} / \mathrm{mol}$; volume molaire $=23,78 \times 10^{-6} \mathrm{~m}^{3} / \mathrm{mol}$; pression de vapeur $=1,43 \times 10^{-5} \mathrm{~Pa}$ à $-39,15^{\circ} \mathrm{C}$; vitesse du son $=3200 \mathrm{~m} / \mathrm{s}$ à $20{ }^{\circ} \mathrm{C}$; chaleur massique $=1230 \mathrm{~J} / \mathrm{kg} . \mathrm{K}$; conductivité thermique $=141 \mathrm{~W} / \mathrm{m} . \mathrm{K}$. Le sodium ne brûle à l'air qu'au-dessus d'une température de $115^{\circ} \mathrm{C}$.

14 Les matériaux des gaines sont étudiés dans les pages 20-21 de la référence [16].

15 L'objectif de toute l'installation des fluides caloporteurs est actuellement de produire de la vapeur d'eau aux plus hautes pressions, températures et débits possibles. À la sortie du générateur de vapeur de Phénix, la température de la vapeur d'eau est d'environ $510^{\circ} \mathrm{C}$, avec une pression de 165 bars à la sortie du surchauffeur, avec un débit de $250 \mathrm{~kg} / \mathrm{s}$ pour chaque générateur de vapeur (voir référence [2], p. 20).
} 
Notons enfin que ces problèmes de transformation de phase, et en particulier l'ébullition du fluide, sont des transitions du premier ordre qui suivent une séquence "germination croissance ». Comme pour tous les phénomènes de ce type, la germination est très souvent hétérogène, commence sur les surfaces, et en particulier pour la germination, sur la surface de la gaine. Cette germination hétérogène dépend fortement de la nature chimique et de la rugosité de surface (voir le document 6.1). L'approche continue classique intègre ces faits par un paramètre empirique intervenant dans le calcul du flux critique conduisant à la crise d'ébullition. Une approche microscopique, qui intègrera de plus I'influence du cisaillement dans le fluide caloporteur, permettra une meilleure compréhension du phénomène. Cette limitation actuelle de l'approche thermodynamique d'équilibre sera reprise dans le paragraphe suivant sur la pression.

\subsection{Pression}

Enfin, la partie de la source froide (condenseur) et la turbine ${ }^{16}$ et ses étages de travail et de circulation doivent être à haute pression (au-dessus de 100 bars), d'où un gradient de pression entre le circuit secondaire de refroidissement et le convertisseur d'énergie. Là encore, la surface métallique séparant ces deux circuits, et au travers de laquelle la chaleur doit passer, est de l'ordre de $400 \mathrm{~m}^{2}$ à $800 \mathrm{~m}^{2}$, suivant les puissances à évacuer dans l'atmosphère.

Cette grande surface d'échange est illustrée pour les refroidisseurs des grandes tours, transformant cette chaleur en vapeur d'eau.

\section{Les circuits des caloporteurs constituent-ils des systèmes thermodynamiques?}

Le cahier des charges du fluide caloporteur doit traduire l'obtention de travail thermodynamique, à partir de la chaleur et de sa température, " couple thermique » produit par la chaudière nucléaire, au travers des gaines (voir diapositives 12 et 14 du document 1.1, diapositives 14 et 27 du document 3.1, diapositive 5 du document 4.2 et diapositives 10, 11 et 12 du document 5.3). Pouvons-nous ajouter sans précaution rigoureuse I'adjectif «thermodynamique »? I| faudrait pour cela définir un système convenable (c'est-à-dire avec des frontières bien définies et des conditions aux limites à ces frontières, et où tant le travail que la chaleur initiale soient bien définis) qui soit lui-même thermodynamique (voir référence [1]) et dans lequel l'évolution au cours du temps puisse être remplacée par un cycle de transformation "stationnaire » et réversible. Un exemple de système peut

\footnotetext{
16 Les caractéristiques métallurgiques des divers projets des turbines à hélium sont examinées en page 23 de la référence [16].
} 
être le convertisseur d'énergie : le fluide recevant la chaleur de la paroi qui le sépare du circuit secondaire entre et parcourt tant la turbine loù se fait la génération de travail) que le condenseur (voir chapitre 9 de la référence [2] et diapositive 12 du document 5.3, diapositives 3, 4, 5, 6, 7, 8, 9 et 10 du document 7.2). La pression du fluide, vapeur d'eau ou autre gaz, y est d'environ 100 à 180 bars (avec une température d'arrivée d'environ $550{ }^{\circ} \mathrm{C}$ - voir le tableau sur la diapositive 3 du document 7.2).

Avec les caractéristiques thermiques que nous venons de citer, une mise côte à côte des valeurs numériques des divers caloporteurs est possible. Elle est faite à la diapositive 5 du document 3.1 en ce qui concerne le sodium et le plomb. Notons que l'énergie potentielle stockée ${ }^{17}$ à $500{ }^{\circ} \mathbf{C}$ est très élevée pour le sodium (10 gigajoules $\left./ \mathrm{m}^{3}\right)$, ce qui est de l'ordre de grandeur de l'essence et $1,1 \mathrm{GJ} / \mathrm{m}^{3}$ pour le plomb, ce qui est relativement peu.

Suivant les critères de sélection entre les divers types de réacteurs, on peut chercher des figures de mérites cohérentes pour les comparer. Cela est effectué, dans le cas des RNR, dans le domaine des caloporteurs possibles et de ceux des REP, dans la diapositive 23 du document 5.1.

\section{2 | Propriétés nucléaires}

\subsection{Radioactivité}

La conception du système caloporteur a, dans son cahier des charges, l'objectif $d$ 'arrêter toute fuite de radioactivité produite dans le cœur du réacteur. Des normes maximales sont fixées pour ce faire. Elles sont respectées passivement en fonctionnement nominal, en maintenance et dans les arrêts de la réaction en chaîne, en chargement et déchargement. Un exemple de cette radioactivité provient du phénomène suivant. Quand le

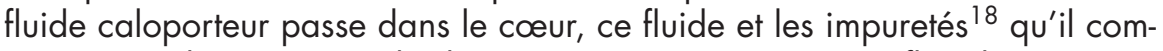
porte (voir diapositive 9 du document 2.2) sont soumis au flux de neutrons, rayons gamma, etc., avec leurs spectres des énergies cinétiques, les réactions

17 Cette énergie caractérise l'énergie pouvant, sans apport extérieur, disperser les noyaux radioactifs des éléments présents dans le système caloporteur ou dans les circuits adjacents. C'est donc une mesure caractérisant une menace éventuelle : voir diapositive 6 du document 3.1. Notons que l'énergie potentielle stockée par le sodium à $500{ }^{\circ} \mathrm{C}$ est plus petite que celle de l'eau (environ $22 \mathrm{GJ} / \mathrm{m}^{3}$ ). Ceci est dû à la décomposition en hydrogène et oxygène de l'eau à haute température.

18 Ceci comporte les migrations de gaz de fission, de tritium, etc., à travers la gaine. Pour les produits de corrosion de l'ensemble du circuit primaire du caloporteur, voir les diapositives 21 , 22,23 et 24 du document 2.2 . 
nucléaires possibles à ces énergies, etc. (voir ces réactions de neutrons sur le sodium dans les diapositives 7 et 13 du document 2.2).

Les sections efficaces de capture des neutrons en fonction de l'énergie cinétique de ceux-ci sont connues, avec des marges d'incertitude (par exemple dans la diapositive 4 du document 4.1 , pour le sodium, les isotopes 39 et 41 du potassium et du lithium-7). On peut ainsi calculer les activations des fluides caloporteurs.

Il en résulte des spécifications des taux d'impuretés du sodium pour ne pas trop l'activer (voir ces spécifications de pureté du sodium dans la diapositive 8 du document 2.2).

Les matériaux des circuits des fluides caloporteurs peuvent aussi contenir des noyaux qui s'activent par le flux des neutrons. Les spécifications de leurs composants et des traces involontaires de matériaux sont données dans la diapositive 10 du document 4.2 pour divers alliages dans le cas du caloporteur à sels fondus.

La décroissance de l'énergie cinétique des neutrons issus de fission, comme suite à leurs collisions avec les noyaux (sodium, plomb, hélium, graphite et, pour repère, l'eau normale et l'eau lourde) du caloporteur et de leurs circuits, est précisée dans la diapositive 10 du document 3.1.

Par exemple, le fluide caloporteur peut corroder une partie sensible d'une paroi de circuit ou de composant (pale de pompe, matériau des joints d'étanchéité d'une vanne ou d'un palier de pompe ${ }^{19}$, siège de robinet ou soupape, partie porteuse d'un palier, purgeur, filtre, soudure, etc.) ; il en est de même pour les produits d'usure et d'érosion. Des écailles de produits de corrosion peuvent être arrachées par le flot à $500{ }^{\circ} \mathrm{C}$, à $10 \mathrm{~m} / \mathrm{s}$ (voir diapositive $8 \mathrm{du}$ document 2.1, diapositives 9, 21 et 23 du document 2.2).

Le flot du fluide caloporteur peut les emporter dans le cœur (voir diapositives 2, 3 et 4 du document 2.2). Là, ces détritus peuvent être irradiés par les neutrons, les gamma, être transformés chimiquement par le rayonnement, etc. Puis la circulation du caloporteur les déposera dans toutes sortes de pièges, dont les pièges froids (voir diapositive 11 du document 2.2). Ces parties de circuits deviendront radioactives.

Les gaz de fission, fortement radioactifs (krypton-85, xénon-133, etc.) sous haute pression ( 90 bars in fine du taux de combustion), peuvent percoler à travers les endroits affaiblis des gaines (de 600 à $800^{\circ} \mathrm{C}$ ) (voir diapositive 9 du document 3.1) et déboucher dans le circuit du caloporteur. Il en est de même du tritium formé dans les fissions ternaires et les captures

19 Voir la référence [18], dans le cas d’un réacteur de $\|^{e}$ génération, à eau sous pression. 
de neutrons par le bore des absorbants des barres de pilotage, etc. (voir chapitre 11 de la référence [2]).

Le caloporteur peut subir des réactions nucléaires directes. Par exemple, pour le sodium, on peut obtenir les isotopes 24 et 22, de demi-vie respectivement de 14,96 heures et 2,6 ans. Les parois en acier inoxydable des réacteurs nucléaires peuvent produire du fer-55 (2,73 ans) ou du manganèse-54, dont la durée de vie est de 312,2 jours.

\subsection{Réaction en chaîne et réactivité : transport de la radioactivité}

En résumé, le circuit caloporteur doit transférer des mégawatts de chaleur, à haute température, dans le sens source chaude vers source froide en en perdant le moins possible, tout en empêchant la radioactivité de faire le même parcours. Cette contradiction du cahier des charges oblige à des compromis.

Suivant le critère retenu par le concepteur - privilégier les hautes températures ${ }^{20}$, donc le rendement, ou bien augmenter la production de matière fissile (ou diminuer les captures) à puissance fournie donnée, diminuer les manipulations, etc. -, les choix de caloporteurs varient.

\section{Chimie}

Il va de soi que la plupart des liaisons chimiques initiales sont brisées (radiolytic dissociation) et d'autres espèces chimiques favorisées par le rayonnement et la température sont créées, sous l'action des radicaux libres (ces espèces trouvent des plages de survie suffisantes pour compter dans la chimie du caloporteur et de son circuit).

Les interactions entre ce fluide et les parois du circuit entraînent des phénomènes encore à explorer pour mieux les maîtriser (voir les diapositives 11, 13, 14, 19 et 22 du document 2.2). Il en est de même pour tous les contacts caloporteur/surface et zone solide liée du circuit primaire. Le point sur les connaissances relatives au sodium, est effectué dans le document "Corrosion dans les contacts entre sodium et surfaces des circuits caloporteurs " ${ }^{21}$ (diapositives $20,21,22,23$ et 24 du document 2.2 et références $[38,39,40])$.

\footnotetext{
20 Pour les concepts de réacteurs à gaz à très haute température, des matériaux céramique et composite-céramique sont étudiés (voir [17]).

${ }^{21}$ La diapositive 8 du document 2 les résume par « metallic sodium is one of the strongest chemical reducers: $\mathrm{Na} \rightarrow \mathrm{Na}^{+}+\mathrm{e}^{-} »$. Cette diapositive groupe les procédés et les équipements qui ont permis d'y faire face.
} 
La chimie du plomb, dans toutes les interactions de ce fluide caloporteur avec les matériaux des circuits de diverses fonctions, est détaillée dans le document 3.3. Notons le rôle de la teneur en oxygène dans ce fluide, qui conduit à le mesurer en continu et à le traiter pour rester sous une teneur maximale de l'ordre de 0,01 \% (diapositives 11, 12, 13 et 14 du document 3.3).

Ce rôle particulier de la teneur en oxygène sur l'agressivité chimique des fluides caloporteurs vis-à-vis du circuit qui les contient se retrouve dans le cas des réacteurs à sel fondu et est détaillé dans le document 4.2. Notons, que ce soit dans le cas du sodium, du plomb ou des sels fondus, l'importance de la chimie locale sur les phénomènes de corrosion ${ }^{22}$. Le caractère éventuellement localisé d'une corrosion accélérée est un vrai problème d'ingénierie : comment disposer d'un réseau de capteurs locaux informant à tout instant et en service sur la chimie locale?

Un problème commun aux caloporteurs de nature métallique est la possibilité d'une fragilisation des matériaux de surface des circuits par les métaux liquides. Ce phénomène est dangereux par sa rapidité $d^{\prime}$ action et son ubiquité. II ne semble pas être limitant pour le sodium avec les matériaux couramment utilisés dans cet environnement, mais il existe de nombreux cas répertoriés pour le plomb et pour de très nombreux métaux liquides. Le document 6.2 donne un état de l'art de la compréhension de ce phénomène complexe qui associe divers facteurs : physicochimie et mécanique, état de contrainte en fin de fissure et modification des potentiels chimiques sous contrainte, mouillage et pénétration intergranulaire. Le couplage contrainte/ dissolution/propagation au cœur du phénomène est encore très mal compris et ce phénomène pourrait être dirimant dans le cas des solutions innovantes concernant les matériaux utilisés. Tout comme pour la question du contact liquide/solide inerte traitée dans le document 6.1, ce type de contact métal liquide/solide sous contrainte constitue une question fondamentale qui demande à être explorée.

\section{4 | Matériaux}

\subsection{Généralités}

Le choix des matériaux constitutifs des réacteurs dépend fortement du fluide caloporteur. Par exemple, l'eau des réacteurs REP est à $350{ }^{\circ} \mathrm{C}$, sous pression, et subit une radiolyse. La mise sous pression de la cuve conduit alors à utiliser un acier bainitique en épaisseur suffisante, mais la tenue à la corrosion

22 Voir référence [18], p. 35. 
impose de déposer dans cette cuve un revêtement en acier inoxydable. Pour le RNR sodium, la température est plus élevée mais la pression est moindre. L'agressivité chimique est limitée, et un acier inoxydable classique présente à la fois la tenue mécanique et la tenue à la corrosion nécessaires, évitant de recourir pour la cuve à une solution bimatériau.

Pour des raisons de rendement thermodynamique des machines thermiques, on a, dans les dessins successifs des réacteurs à fission, une tendance de fond à augmenter la température de fonctionnement ${ }^{23}$. Cela conduit à augmenter les cinétiques de corrosion, à amplifier les phénomènes viscoplastiques comme le fluage, à modifier les équilibres entre les dommages d'irradiation et les phénomènes de restauration. Pour le cas spécifique des réacteurs à neutrons rapides, une étude approfondie, du point de vue fondamental, de la tenue sous irradiation à fort flux et à haute température ${ }^{24}$ semble de plus en plus nécessaire pour valider ou invalider les solutions innovantes ${ }^{25}$ à l'étude, en particulier pour le gainage, comme pour les aciers ODS26 (Oxide Dispersion Strength) ou les $800 \mathrm{H}$ et $800 \mathrm{HT}$. En effet, la gaine est la barrière la plus sollicitée et la plus cruciale.

Une distinction essentielle entre les composants des divers circuits des caloporteurs concerne la possibilité ou non de les changer pendant les périodes de maintenance ou de réparation. Ce n'est pas le cas de la cuve dans les concepts de réacteurs "piscine » des RNR refroidis au sodium (voir diapositive 2 du document 2.2). Qui dit piscine dit surface libre du sodium et donc aussi atmosphère gazeuse le surplombant (voir diapositive 28 du document 2.2). Des aérosols peuvent en être à l'origine (voir diapositive $29 \mathrm{du}$ document 2.2). II faut donc prévoir un processus pour les supprimer.

Le choix des matériaux de cette cuve est donc « stratégique » pour l'exploitation de ce concept. Le détail en est donné dans le document 7.2. Leur capacité d'inspection et de réparation in situ est exposée dans les diapositives 23 , $24,25,27,29$ et 31 du document 7.2.

Les opérations discontinues, dites " batches " (voir diapositive $5 \mathrm{du}$ document 2.2), de l'exploitation du cœur des réacteurs nucléaires actuels sont liées au taux de combustion maximal acceptable, lui-même commandé par les propriétés des solides des combustibles. Ouvrir un concept annulant

\footnotetext{
${ }^{23}$ Voir la référence [16].

24 Un substantiel exemple d'étude approfondie a été effectué par le programme DRAGON. L'Agence pour l'énergie nucléaire de l'OCDE a édité la totalité des résultats sous forme de documents, puis sur CD-Rom. Un guide général en est la référence [24].

25 Voir les publications du laboratoire Argonne National Laboratory sur ces sujets dans la référence [33].

${ }^{26}$ Voir les références $[25,36]$.
} 
cette contrainte est un objectif considérable, demandant une recherche à très long terme (voir diapositives 2, 4, 5, 6 et 7 du document 4.2).

Un des défis principaux des RNR est d'augmenter le rendement de conversion du cycle thermodynamique, donc de monter en température ${ }^{27}$ la chaleur fournie à l'étage de conversion de la chaleur en travail thermodynamique. $C^{\prime}$ est une piste à explorer dans l'option du caloporteur gazeux. Les températures visées sont citées dans les diapositives 5 et 8 du document 5.3.

II faut être conscient qu'étudier les problèmes scientifiques et techniques des RNR, c'est étudier des concepts qui devraient être utilisables dans le monde entier, avec toutes sortes d'infrastructures, en particulier les transports, I'habitat, etc. Remplacer les fluides combustibles actuels, fossiles, par des substituts chimiques demanderait de la chaleur utilisable pour toutes ces applications, donc de l'énergie à haute température. C'est dire que la production d'énergie ne viserait pas seulement une conversion sur place de la chaleur en électricité, mais aussi la fabrication de combustibles liquides utilisables dans les conversions distribuées que sont les moteurs des transports de toute nature. C'est souligner une fois de plus que l'un des objectifs de la génération IV pourrait bien être d'obtenir la chaleur, avec un niveau très élevé de température ${ }^{28}$ (voir les documents 5.1, 5.2, 5.3 et la référence [7]).

Un des thèmes ${ }^{29}$ de recherche ${ }^{30}$ majeurs est l'étude du comportement des divers aciers des circuits en contact avec les fluides caloporteurs (voir pour le sodium la diapositive 14 du document 2.3, pour le plomb les diapositives $7,8,9,10,11,12,13,15,16,18,20$ et 23 du document 32 , pour I'hélium les diapositives 12, 14, 16, 18, 19, 20, 22, 24, 25 et 27 du document 5.2 et pour les sels fondus les références $[22,23])$.

\subsection{Construction mécanique et contrôle des composants}

Une carte des composants et des processus à l'intérieur du réacteur est analysée dans l'encadré 4 en pages suivantes.

\footnotetext{
27 Voir un exemple d'installation pour pratiquer des essais dans la référence [20].

28 Voir la référence [26].

29 Une revue des divers thèmes scientifiques des matériaux de ces quatre concepts de réacteurs est dans le tableau page 11 de [19]. Signalons la liste des références à la page 14 de [19].

30 Un des outils majeurs de la recherche en matériaux est l'utilisation de codes de calcul tels que CALPHAD (méthode et base de données : voir [28]) et, à partir de celui-ci, MATCalc (the materials calculator: http:/matcalc.tuwien.ac.at; voir [29]) pour étudier la cinétique des précipitations dans l'acier. Un programme de modélisation multiéchelles, valable pour les matériaux de la fusion autant que pour ceux de la fission, est exposé dans la référence [37].
} 


\section{Encadré 4 - La topographie du réacteur ${ }^{31}$}

Un carrefour de fonctions, de matériaux, de composants, d'espèces chimiques, nucléaires, hydrauliques, thermodynamiques ${ }^{32}$

Nous avons vu qu'une partie du circuit caloporteur est à l'intérieur du réacteur $^{33}$ nucléaire. Mais $\mathbf{d}^{\prime}$ autres fonctions y sont également placées.

Régime nominal (voir figures des diapositives 2, 3 et 4 du document 2.2) :

- la réaction en chaîne et donc l'économie des neutrons ;

- le contrôle de la réactivité tant par des dispositifs absorbants mobiles que par des compensations du taux de combustion (voir chapitre 17 de la référence [2]) ;

- le débit de sodium primaire dans le cœur (par exemple, de Phénix) est d'environ $2800 \mathrm{~kg} / \mathrm{s}$ (référence [2], p. 20) à comparer au débit de sodium du circuit secondaire $(740 \mathrm{~kg} / \mathrm{s})$ et à celui du générateur de vapeur $(210 \mathrm{~kg} / \mathrm{s})$;

- I'évacuation de la puissance résiduelle ${ }^{34}$ pendant les manutentions diverses (voir diapositive 3 du document 2.1 et chapitre $V$ de la référence [2]) ;

- le guidage des filets de fluide caloporteur et l'organisation de la turbulence dans le cœur (il y a environ 800 tonnes de sodium dans le circuit primaire de Phénix, et quelques milliers pour une taille de 1 GWe, comme Superphénix) ;

- le chargement des éléments combustibles neufs et la manutention et le lavage des combustibles usés (voir la diapositive 3 du document 8.1 et les deux figures de la page 203 de [2]) ;

- le déchargement des combustibles usés (voir référence [2], figure page 16) ;

${ }^{31}$ Voir les plans schématiques des réacteurs à fluides caloporteurs en sodium dans les diapositives 2, 3, 4 et 13 du document 2.2, diapositive 6 du document 2.3, au plomb dans les diapositives 14,15, 16 et 17 du document 3.1, à I'hélium dans les diapositives 17 et 18 du document 5.1 et la diapositive 11 du document 5.3 et au sel fondu dans les diapositives 2, 3, 4,5 et 12 du document 4.2 .

32 Pour le cas important de l'ingénierie des hautes températures dans la cuve du réacteur, voir la référence [35].

33 Voir la référence [41].

${ }^{34}$ Les dispositifs d'évacuation de la puissance résiduelle, dans le cas du caloporteur sodium, sont décrits par le schéma de la diapositive 7 du document 2.1. 
- les instruments de mesure, neutroniques, thermodynamiques, chimiques, nucléaires, etc. ;

- I'inspection des gaines et des autres composants des circuits des caloporteurs (voir référence [2], pp. 191-192 de Joël Guidez) ;

- la compensation de la diminution progressive du matériau fissile (due à une réaction en chaîne), grâce à la destruction graduelle d'un corps absorbant de neutrons, pour maintenir exactement la criticité ;

- les dispositifs de manutentions pour les diverses opérations ;

- I'inspection de la cuve.

Plus généralement, toutes les manutentions du fluide caloporteur métallique, de ses circuits quand ce fluide est présent, se font dans des conditions d'opacité vis-à-vis de la lumière. On a recours alors à des images par ultrasons et par électromagnétisme (voir par exemple les diapositives 17 et 18 du document 2.2, diapositives 15 et 16 du document 2.3 et chapitre 17 de la référence [2] ainsi que la figure p. 191 indiquant le bilan des inspections techniques effectuées dans le réacteur Phénix).

\section{Régime accidentel :}

- I'évacuation de la puissance résiduelle (voir diapositive 6 du document 1.3) ;

- la canalisation des détritus éventuels de fusion du cœur (" corium ») ;

- le guidage des filets de fluide caloporteur et l'organisation de la turbulence ;

- les manutentions en régime d'incident ou d'accident;

- I'encombrement et les inspections?

Peut-on diminuer, autant que faire se peut, les éventuels couplages entre ces fonctions, lors d'un écart au régime nominal du point de vue topographique, autant que faire se peut? Peut-on alléger la foule des phénomènes qui s'amassent dans ce réacteur? Un exemple est la tentative d'en retirer la fonction chargement-déchargement.

Les opérations de démantèlement des divers concepłs de réacteurs liés au système caloporteurs sont-elles significativement différentes ? La réponse est clairement positive pour une fonction importante. La maturité scientifique et technique ${ }^{35}$ des systèmes de refroidissement avec les divers

35 Un exemple de maturité scientifique et technique est celui du caloporteur sodium. Les voies de recherche et développement sont clairement identifiées. Voir la diapositive 14 du document 1.1. 
caloporteurs est fort différente. Certains des métaux liquides ont été utilisés pendant plusieurs décennies ${ }^{36}$, avec plusieurs centaines d'années de fonctionnement cumulé. $D^{\prime}$ autres catégories de fluides caloporteurs, très stables du point de vue du réglage de la réactivité et du taux de combustion maximal, sont étudiées. Cela nécessite des travaux en chimie et physicochimie [37] sous rayonnement, donc une recherche et développement à étendre sur le long terme.

\section{5 | Risques}

Nous avons passé en revue quelques caractéristiques des RNR liées aux fluides caloporteurs. La question globale de la sûreté des réacteurs de génération IV dépasse le cadre de cet ouvrage. Un panorama général a été effectué par [3, 30]. On peut en revanche étudier quelques composantes des éventuels risques rencontrés en liaison avec les fluides caloporteurs pour lesquels une expérience substantielle existe dans de nombreux pays et, en particulier, pour les réalisations et exploitations les plus longues comme le refroidissement au sodium les plus anciens. Les orientations pour résoudre les problèmes de sûreté des RNR à caloporteurs sodium, aux Etats-Unis, sont traitées dans la référence [39]. Une méthodologie générale est exposée dans la référence [32]. Toutefois, quelques questions de méthodes restent ouvertes. Par exemple, quel sens cela a-t-il de vouloir comparer les risques associés à des caloporteurs différents, ayant une réactivité chimique ou un pouvoir corrosif aussi contrasté que le sodium, le plomb, le sel fondu, I'hélium et l'eau?

Peut-on évaluer les éventuels risques des systèmes de refroidissement par caloporteur avec une même échelle où l'on positionnerait les risques associés au cycle du combustible, à l'exploitation des centrales, au démantèlement, à l'entreposage et au stockage des déchets radioactifs? Au fil des exposés du séminaire, quelques observations ou questions se présentent.

Première observation: dans l'optique d'une réflexion globale sur les risques, tant des réacteurs, du cycle de combustible, des transports, etc., et jusqu'au retour à des terrains libres d'emploi, peut-on séparer et donc énumérer des composantes de risque, et voire même les comparer ? Un tel exemple de variable libre est constitué par le cahier des charges (voir les diapositives 9, 10,11 et 12 du document 1.3 et les diapositives 4,5 et 7 du document 4.1).

Deuxième observation : peut-on associer à chacune des composantes du risque un index numérique qualifiant l'enveloppe des réalisations des dégâts qu'il peut entraîner ? Un exemple en est fait pour les dégagements d'énergie chimique pouvant abîmer les équipements par la notion de stored potential

36 Voir la référence [21]. 
energy, définie et développée par Toshinsky, à ICAPP 2011 (voir la diapositive 6 du document 3.1, pour le sodium, le plomb et le cas de référence des REP). Toutefois certains risques sont en corrélation, donc non indépendants.

Troisième observation : plusieurs décompositions des composantes des risques liés au caloporteur sont loisibles ${ }^{37}$.

Quatrième observation : peut-on faire des enveloppes de tous les risques plausibles, qu'ils soient dépendants ou interdépendants? Un exemple en est le rôle singulier et majeur de l'enceinte de confinement de tous les circuits de refroidissement radioactifs et de son emplacement.

\section{6| Bilan}

En terminant cette introduction aux exposés relatifs aux propriétés physiques et chimiques des fluides caloporteurs des RNR, nous constatons qu'un travail scientifique considérable a été effectué dans le monde, dont une partie substantielle en France. L'objectif de ce séminaire était d'en faire le point et d'identifier les défis scientifiques majeurs, vus avec les connaissances d'aujourd'hui. Ces défis, comparés aux fluides de refroidissement des REP, sont liés à :

- de plus hautes températures, tant pour les éléments combustibles que pour les matériaux de structure des circuits de refroidissement afin d'améliorer le rendement thermodynamique. Ceci est loisible car le verrou que constitue l'ébullition de l'eau (un changement de phase, le grand problème des fluides de refroidissement des réacteurs) a disparu dans les RNR ;

- de plus hautes fluences de neutrons pour compenser les faibles sections efficaces de fission dans la gamme des centaines de keV;

- de plus grands dégâts d'irradiation, dus au spectre d'énergie cinétique élevé des neutrons ;

- des matériaux de gaine sans la contrainte des absorptions de neutron, mais soumis à de plus hautes températures que dans les REP, le taux de combustion plus élevé que dans les REP et donc la constitution d'une pression interne due aux gaz de fissions, à l'hélium, etc. ;

37 Citons le mode de comparaison exposé dans la diapositive 13 de 2.1 pour des fluides caloporteurs métalliques. 
- des puissances spécifiques du cœur plus élevées que dans les REP, ce qui diminue la taille d'ensemble de la source de chaleur, mais est une contrainte sur le fluide de refroidissement.

\section{Références}

[1] Sauvage J.F. (2009) Retour d'expériences Phénix, Superphénix, note EDF, ENDPJU090002A.

[2] Guidez J. (2013) Retour d'expérience Phénix, CEA.

[3] Dautray R., Brézin É. et al. (2012) Science of nuclear safety postFukushima, C. R. Physique 13.

[4] Atkins P., de Paula J. (2012) Atkin's Physical Chemistry; Equilibrium; The first law (pp. 44-93), The second law (pp. 94-135), Physical transformations of pure substances (pp. 136-155) ; Simple mixtures (pp. 156208); Chemical equilibrium (pp. 209-246), Thermodynamics, 9th edn. Oxford University Press, Oxford.

[5] Bailly H., Ménessier D., Prunier C. (année ?) Le combustible nucléaire des réacteurs à eau sous pression et des réacteurs à neutrons rapides. Eyrolles, collection CEA/Synthèses.

[6] Weinberg A., Wigner E. (1955) The Physical Theory of Neutron Chain Reactor. University of Chicago Press.

[7] Weber M., Duncan R., Shivers Gonzales M. (2013) Four technologies and a conundrum. The glacial pace of energy innovation, Issues in Science and Technology Winter, 79.

[8] Brézin É. (2002) Du combustible nucléaire aux déchets : recherches actuelles. C. R. Physique 3.

[9] Anzieu P. et al. (2006) Les réacteurs nucléaires à caloporteur gaz, CEA/ DEN-direction de l'énergie nucléaire.

[10] Académie des sciences (2011) L'accident majeur de Fukushima. EDP Sciences, Les Ulis.

[11] Académie des sciences (2012) La recherche scientifique face aux défis de l'énergie. EDP Sciences, Les Ulis.

[12] Nikolayev V., Beysens D., Garrabos Y., Lecoutre C., Chatain D., Pichavant G., Cariteau B. (2010). Physique de l'ébullition, INAC - CEA. 
[13] Atkins P. (2007) Four Laws that Drive the Universe (Thermodynamics). Oxford University Press, Oxford.

[14] Derrida B., Gaspard P., Van Den Broeck C. Guest editors (2007) Work, dissipation, and fluctuations in non equilibrium physics, C. R. Acad. Sci. 8.

[15] L'École "Sodium », CEA Cadarache, Département de technologie, www.cadarache.cea.fr

[16] Allen T., Burlet H., Nanstad R.K., Samaras M., Ukai S. (2009) Advanced structural materials and cladding, MRS Bulletin 34, 20.

[17] Bonal J.P., Kohyama A., van der Laan J., Snead L. (2009) Graphite, ceramics and ceramic composites for high-temperature nuclear power systems, MRS Bulletin 34, 28.

[18] Cabet C., Jang J., Konys J., Toryorelli P.F. (2009) Environmental degradation of materials in advanced reactors, MRS Bulletin 34, 35.

[19] Guerin Y., Was G., Zinkle S. (2009) Materials challenges for advanced nuclear energy systems, MRS Bulletin 34, 10.

[20] Hvasta M., Nollet B., Anderson M. (2012) Design and development of a high temperature sodium compatibility material testing facility, University of Wisconsin.

[21] Grandy C. (2012) Advanced reactor concepts research and development include sodium technology R and D, Argonne National Laboratory, Nuclear Engineering Division.

[22] Corwin W., Wilson D. (2010) Fluoride salt cooled high temperature reactor (FHR) materials challenges, FHR Workshop, Oak Ridge, TN.

[23] Haubenreich P.N., Engel J.R. (1970) Experience with the molten salt reactor experiment, Nuclear applications and technology 8, 118.

[24] Shaw E.N. (1983) Europe's nuclear power experiment - History of the OECD Dragon project, Oxford Pergamon et NEA Data Bank, Scientific content, nuclear energy agency, www.nea.fr/dbprog.

[25] Rouffié A.L., Fournier B., Wident P., Ziolek L., Delabrouille F., Tanguy B., Crépin J., Garat V., Pineau A. (2012) Influence of the microstructure on the fracture mechanisms of ODS ferritic steels, Kazan.

[26] Hollner S., Fournier B., Le Pendu J., Cozzika T., Tournié I., Brachet J.C., Pineau A. (2010) High temperature mechanical properties improvement on modified $9 \mathrm{Cr}-1 \mathrm{Mo}$ martensitic steel through thermomechanical treatments, Journal of nuclear materials 405, 101. 
[27] Hollner S., Fournier B., Mayr P., Pineau A. (201 1) Thermomechanical improvement of high-temperature mechanical properties of 9-12\% martensitic steels for nuclear applications, Proceedings of ICAPP, 11314.

[28] Saunders N., Miodownik A.P. (1998) CALPHAD, A Comprehensive Guide. Pergamon Materials Series.

[29] MATCalc the materials calculator, http:/matcalc.tuwien.ac.at, Software project for computer simulation of phase transformations in metallic systems, Constrained and unconstrained phase equilibria; precipitation kinetics; long range diffusion; simultaneous diffusion and precipitation; phases transformation/moving phase boundaries; lattice metropolis and kinetic Monte Carlo, The thermodynamic foundation is CALPHAD. An alternative software, using the same principles is DICTRA, http:// www.thermocalc.com/DICTRA.htm, developped by the Swedish school.

[30] Panorama des filières de réacteurs de génération IV. Appréciations en matière de sûreté et de radioprotection, rapport IRSN/DG/201200002 du 21 mars 2012.

[3 1] Zhu Y., Granick S. (2002) Limits of the hydrodynamic no-slip boundary condition, Phys. Rev. Lett.. 88, 106102.

[32] Bensoussan A. (2012) Risk management: An overview, Presentation to AEC and NTHU, Taiwan.

[33] Hatesan K. (2012) Corrosion and mechanics of material, Argonne National Laboratory, note interne.

[34] Technologies des réacteurs à métaux liquides, CEA Cadarache, Département de technologie nucléaire, disponible sur : http://www-cadarache. cea.fr/fr/activities/fission/dtn/Plateformes/sodium/plateforme_S.

[35] Survey on basic studies in the field of high temperature engineering, 27-29 September 1999, JAERI and Nuclear Energy Agency/OECD.

[36] Boutard J.L. (2010) ODISSEE, aciers renforcés par dispersion d'oxydes : problèmes de base posés par la mise en œuvre et l'utilisation sous le haut flux de neutrons rapides des réacteurs de fission et de fusion, CEA/ DEN/DMN.

[37] Willaime F. (2008) Modélisation multi-échelles des matériaux sous irradiation ; récents progrès et perspectives, CEA Saclay, Service de recherche de métallurgie physique, Workshop ITER.

[38] Anzieu P. (2011) Sodium technology and safety; R and D program in support to Astrid, CEA/DEN, GIF-INPRO meeting AIEA Vienna, Austria, November 30. 
[39] Approaches to resolve safety issues related to sodium as a fast reactor coolant, Federal State Unitary enterprise, State scientific centre of the russian federation-institute for physics and power engineering, named after A.I. Leypunsky, Second joint GIF-IAEA/INPRO workshop on safety aspects of sodium-cooled fast reactors, 30 November-1 st December.

[40] Wigeland R., Stanculescu A. (2011) SFR safety approach in the United States, Second joint GIF-IAEA/INPRO workshop on safety aspects of sodium-cooled fast reactors, 30 November-1 st December 2011.

[41] Grandy C. (2011) An overview of U.S. SFR design concepts. Second joint GIF-IAEA/INPRO workshop on safety aspects of sodium-cooled fast reactors, 30 November-1 st December.

[42] Martin G. (2005) Driven alloys, CEA and first laureate of the Rhine Ruhr international materials award.

[43] Mahelle P., Thuillier R. (2013) Renforcement de la sûreté des joints d'arbres des groupes motopompes primaires. Revue générale nucléaire 1, 54.

[44] Durran D.R., Frierson D.M.W. (2013) Condensation, atmospheric motion, Physics Today 66 (4), 74.

[45] Balian R. (2007) From Microphysics to Macrophysics, Methods and Applications of Statistical Physics, volumes 1 and 2, Springer Verlag, 9th edition. 